\title{
A system-level investigation into the pharmacological mechanisms of flavor compounds in Maotai liquor
}

\section{Zhigang Liu ( $\nabla 784343646 @ q q . c o m$ )}

Shenzhen University https://orcid.org/0000-0002-4896-4453

\section{Wei Zhou}

Shenzhen University

\section{Ziyi Chen}

Shenzhen University

Guohao Zhang

Shenzhen University

Research article

Keywords: Flavor compounds, Maotai liquor, System-level investigation, Complex mechanism, Human health

Posted Date: March 3rd, 2020

DOl: https://doi.org/10.21203/rs.3.rs-15719/v1

License: (9) This work is licensed under a Creative Commons Attribution 4.0 International License. Read Full License 


\section{Abstract}

Maotai-flavor liquor, one of the world's famous natural fermentation food product plays a significant role in traditional Chinese culture and people's daily lives for a long time due to its good taste. However, the pharmacological activities of flavor compounds in Maotai liquor has not been fully elucidated. In answering this question, a system-level analysis was developed by combining in silico ADME evaluation, multi-target prediction, $\mathrm{GO}$ enrichment analysis, network pharmacology technology, pathway analysis, as well as experimental verification to elucidate the pharmacological effects of flavor compounds in Maotai liquor. Finally, 55 active compounds and 80 targets were identified to interpret the pharmacological effect of the flavor compounds. Moreover, the key active compounds was verified by in vitro experiments to validate the reliability of our approaches. Our study provides a novel integrated strategy to comprehensively understand the pharmacological activities of complex components in Maotai liquor.

\section{Introduction}

Chinese liquor is one of the oldest distilled alcoholic beverages in the world, which is normally fermented from grains, mainly containing corn, rice, wheat, and sorghum. Except for being a useful business tool, Chinese liquor plays a significant role in the traditional culture of China and people's daily lives. Since fermented alcoholic drink had considerable social, religious, and medical status from ancient times until today. In particular, the relationship between Chinese liquor and healthy aspects. Chinese people believe that liquor is a healthy product for a long time and moderate drinking is good for health. The Chinese Materia Medica compiled by Li Shi-Zhen recorded that moderate drinking of liquor may relieve the feeling of cold, eliminate fatigue and phlegm dampness. In addition, some clinical studies on Chinese liquor revealed that consumption of liquor in small volumes every day may play a role in various human diseases. For example, it has been reported that adequate drinking of Liquor could decrease platelet aggregation, reduce the formation of blood clots and accelerate blood circulation, so as to enhance the functioning of cardiovascular and circulatory systems [1]. Evidence from another research suggested that moderate daily liquor intake decreases concentration of uric acid in serum and participates in the prevention of Alzheimer's disease [2].

Maotai-flavor liquor, known as "National wine" in China, is a symbolic drink of China on a par with whisky of Scotland and brandy of France. As one of the few examples of liquor, the production of Maotai is distilled from traditional solid-state fermentation that involves the interaction of several distinctive microbial communities, the unique traditional technology and the advanced scientific process, leading to the special flavor and aroma, sweet and refreshing of the liquor. Some studies suggested that Maotai liquor has been found to be beneficial to human health. For instance, the epidemiology survey result showed that consuming Maotai liquor $150 \mathrm{~g}$ for ten years daily did not cause liver diseases, but could help protect human health [3]. The evidence has been proved that Maotai liquor might interfere with cancer formation by inducing anti-oxidative metallothioneins (MTs), which could remove free radicals and participate in an array of protective responses [4]. 
Despite the longstanding belief that moderate consumption of Maotai liquor is good for health and safe, the relationship between Maotai liquor and health is very complex. For one reason, the brewing technology of Maotai liquor is a very intricate process involving the interaction of various complicated microbial community to form a complex fermentation system and eventually leads to the flavor compounds formation and Maotai liquor production. Furthermore, although analytical chemists have already succeeded in identifying the flavor compounds in Maotai liquor, the active ingredients, target proteins in the human body, and mechanisms of the interaction between them are still unclear. Therefore, it is urgent to establish a comprehensive method for uncovering the active compounds, pharmacological targets and their molecular interaction mechanism, so as to clarify the relationship between flavor compounds in Maotai liquor and health at a system level.

Fortunately, systems pharmacology is an emerging interdisciplinary area employing systems biology, pharmacology, biochemistry and genomic to develop a comprehensive framework for dissecting the mechanisms of multi-component and interrelationships of complex biological systems by using computational or mathematical modelling and experiments techniques. Systems pharmacology is likely to be a new powerful approach that integrates pharmacokinetic data (ADME characteristics of a drug), multiple targets prediction and validation, GO enrichment analysis, network pharmacology technology, pathway analysis, as well as experimental verification to explore the drug actions across multiple scales of complexity from molecular to cellular, tissue and organism levels. The successful application of systems pharmacology is not only helpful to promote systems-level understanding of the mechanism of drug action and therapeutics, but also critical for facilitating the drug discovery and development of complex disease therapy. In recent years, systems pharmacology plays a significant role in the research of herbal and western medicines, including predicting bioactive drug ingredients and potential targets, revealing pharmacological synergy and therapeutic mechanisms, resolving the Chinese tradition medicine theory and identifying multiple toxin-target interactions based on chemical, genomic, and toxicological data and so on $[5,6]$.

Therefore, in this study, we provide an integrated strategy by combining active compounds screening, multiple compound targets prediction, gene ontology enrichment analysis and network pharmacology techniques to systematically investigate the active substances and pharmacological mechanisms of Maotai liquor. Knowledge of the multiple molecular mechanisms of flavor compounds in Maotai liquor based on systems pharmacology not only facilitates the further understanding of the pharmacological effect of flavor compounds, but also opens up new ideas to discover the active compounds that may contribute to human health. The framework of systems pharmacology approach was shown in Fig. 1.

\section{Materials And Methods}

\subsection{Construction of data sets}

A total of three hundred and ninety six compounds, including twenty nine organic acids, sixty alcohols, one hundred and forty-four esters, forty-nine ketones, thirty nine aldehydes, twelve phenols acetals, sixty- 
three nitrogen-containing and sulfur-containing compounds were collected as the important flavor compounds of Maotai liquor from the references published in the past years [7-11]. Most of them exist widely in nature and are also found in other Chinese liquors, but several compounds such as 1-propanol, trimethyl pyrazine, 2-furanmethanol, 2-heptanone, 1-pentanol and tetramethylpyrazine are uniquely identified in Maotai liquor [10]. The structures of these compounds were collected from The PubChem project (https://pubchem.ncbi.nlm.nih.gov/). All the compounds were saved in mol 2 format which will be used in the next step of research work.

\subsection{Active compounds screening}

ADME (absorption, distribution, metabolism and excretion) evaluations of a candidate chemical lead(s) are critical procedures to filter active compounds with favorable pharmacokinetic properties and potential biological effect [12]. Because of the disadvantages that conventional experimental approaches are timeconsuming, laborious and high-costy, in silico approaches are being successfully applied to ADME prediction for the screening and analysis of bioactive compounds in pharmaceutical research. Therefore, in our study, four ADME-systems evaluation models, which integrated lipophilicity (logP, logarithm of octanol-water partition coefficient), oral bioavailability (OB) prediction, Caco-2 permeability and blood brain barrier (BBB) were employed to evaluate the potential bioactive compounds of Maotai liquor.

\section{Lipophilicity}

Molecular lipophilicity is another key physicochemical parameter with the definition of the partition coefficient $P(\log P)$. It plays a significant role in determining ADME properties as the quality and pharmacologic effect of compound can be improved by controlling the lipophilic within an optimum range [13]. In this work, lipophilicity was calculated by ALOGPS 2.1 program, which established a computational algorithm for lipophilicity based on 64 neural networks. The model was built based on 12,908 experimental log $\mathrm{P}$ values of the compounds obtained from PHYSPROP database and 75 input parameters, and showed good predictability with root mean square $=0.49$ and standard mean error $=0.26$ [14]. Finally, the values range of $\log P$ is between 1 and 5 according to the Lipinski's rule of five [15].

Oral bioavailability

$\mathrm{OB}$ is one of the most vital pharmacokinetic parameter among $A D M E$ properties which indicates ratio of oral dose of a given compound delivering to the circulatory system in the body [16]. In the present study, in order to screen compounds which have poor potential pharmacological effects, a reliable model OBioavail 1.1 was introduced to calculate the OB values of flavor compounds in Maotai liquor [17]. This model was constructed by using multiple linear regression (MLR), partial least square (PLS) and supportvector machine regression (SVR) methods based on 805 structurally diverse drug molecules. The SVR model exhibits excellent performances with its determination coefficient $R^{2}=0.80$ and the standard error of estimate $\mathrm{SEE}=0.31$ for the training set, the regression coefficient $\mathrm{Q}^{2}=0.72$ and the standard error of prediction SEP $=0.22$ for the test set. In this work, the threshold of OB value was set to $60 \%$ since the average oral bioavailability was $30 \%$ with $10-50 \%$ changeability on the basis of clinical studies. The 
following two careful considerations describe the design rules of the threshold determination: (1) to obtain as much information as possible from Maotai liquor using the least number of molecules; (2) to reasonably interpret the model by the reported pharmacological data.

\section{Caco-2 permeability}

The absorption of orally administered compounds across the intestinal epithelial barrier is an important characteristic for potential drugs, which determines the ability of human absorption and eventually influence its bioavailability. Caco-2 cells have been widely applied to predict intestinal absorption in humans on account of their unique capability to model compound absorption properties [18]. Many in silico models using in vitro Caco-2 permeability have been widely used in pharmaceutical research to understand compound's intestinal absorption and transport processes [19]. Therefore, in our present study, a robust in silico Caco-2 permeability prediction model was employed to evaluate Caco-2 intrinsic permeability and identify highly permeable compounds. This model was built based on 100 drug compounds and exhibited a satisfactory statistical result $\left(R^{2}>0.8\right)$ [20]. Finally, we set the critical value of Caco-2 permeability to -0.4 due to the fact that compounds with Caco- $2 \leq-0.4$ were not permeable.

BBB penetration

Blood-brain barrier is a selective permeability barrier that separates central nervous system (CNS) from the systemic circulation in order to prevent the entrance of potentially harmful substances into the brain [21]. The prediction models of BBB permeability are regarded as a useful tool in screening candidate compounds for disease management. Therefore, in this section, a reliable BBB model was involved to evaluate the abilities of compounds to pass through the BBB by simple diffusion [22]. The model was developed with 190 related but chemically diverse compounds by using partial least squares discriminant

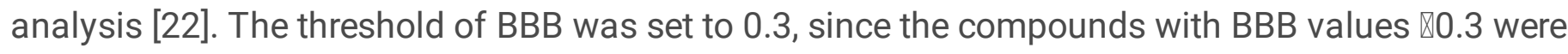
considered as strong penetrating.

\subsection{Target prediction}

Systematically determining of compound-target interactions is a key step for elucidating the mechanisms of biological phenotypes elicited by bioactive compounds. Thus, in order to identify the targets of screened active compounds, several in silico prediction methods enriched with chemogenomic, integrated data-mining, pharmacological and statistical methods were involved as following: (1) The Engerprint Similarity Ensemble Approach (SEA, http://sea.bkslab.org/) based on the chemical similarity of their ligands and information integration method STITCH (http://stitch.embl.de/) that combined the available knowledge on compound-protein interactions were implemented to predict the potential targets of active compounds. In addition, a chemogenomic integrated model (DTpre) that integrated the chemical, genomic and pharmacological information constructed by support vector machine (SVM) and random forest (RF) was used to identify the potential compound-target interactions [23]. DTpre model displayed good performance of predicting the interactions between compounds and targets with concordance of $82.83 \%$, sensitivity of $81.33 \%$, and specificity of $93.62 \%$, respectively. (2) The text mining methods, 
including HIT (Herbal Ingredients' Targets database, http://lifecenter.sgst.cn/hit/), TTD (http://bidd.nus.edu.sg/group/ttd/) and DrugBank, in which data supported by published literatures were applied to obtain more accurate compound-target interactions. (3) Predicted target profiles obtained from the previous two steps were further subjected to TTD, PharmGkb (http://www.pharmgkb.org), UniProt database (http://www.uniprot.org/) and Comparative Toxicogenomics Database (CTD, http://ctdbase. org/) to mine target-related diseases to further investigate the relationships between target proteins and diseases.

\subsection{Network construction and analysis}

In order to better explore the complex relationships among active compounds, target proteins, biological pathways and diseases, two types of networks are established: 1) Compound-Target-Disease network (CT-D network). The network analysis was performed to better explore the comprehensive pharmacology effects of Maotai liquor. The C-T-D network was generated by linking the active compounds, potential targets and their related disease of Maotai liquor, which aimed to systematically elucidate the multi-target and multi-function feature of the active compounds that contribute to diseases. 2) Target-Pathway network (T-P network). The canonical pathway information of targets was enriched from DAVID database (https://david.ncifcrf.gov/) for the purpose of constructing a target-pathway bipartite graph which uncovered the interactions of targets and their related pathways. All the visualized networks with their fundamental topological properties were constructed and analyzed by using Cytoscape 3.2.1, an open source software for visualizing, integrating, modeling and analyzing the biological data interaction networks [24].

\subsection{Experimental verification}

Cell culture and chemical treatment

ANA-1 cell line was obtained from Stem Cell Bank, Chinese Academy of Sciences (Shanghai, China) and cultured in Roswell Park Memorial Institute (RPMI) 1640 (Gibco, NY, USA). DC 2.4 cell line was purchased from American Type Collection Culture (ATCC) and cultured in Dulbecco's modified Eagle's medium (DMEM, Invitrogen, Carlsbad, CA, USA). The medium was supplemented with $10 \%$ fetal bovine serum (FBS, Invitrogen, Carlsbad, CA, USA) and $50 \mathrm{U} / \mathrm{ml}$ penicillin/streptomycin (Gibco, NY, USA). Cells were incubated at $37^{\circ} \mathrm{C}$ with $5 \% \mathrm{CO} 2$.

Compounds cedrol, benzothiazole, tetramethylpyrazine and 3-pentanol (purity $\mathbb{8} 9 \%$ ) were purchased from Aladdin Reagent Database, Inc. (Shanghai, China) and dissolved in dimethyl sulfoxide (DMSO). Linearly growing cells were trypsinized and $1.2 \times 10^{6}$ cells were seeded into 6-well plates. After 24 hours, cells were pretreated with individual chemicals at various concentrations for 2 hours followed by stimulation with lipopolysaccharide (LPS, $1 \mathrm{ug} / \mathrm{mL}$ ) for another 6 hours or 24 hours to detect the expression level of RNA and protein, respectively.

Real-time PCR and western blot 
Total RNA was extracted using TRIzol reagent (Invitrogen, USA) according to the manufacturer's instruction. $0.5 \mu \mathrm{g}$ of total RNA was used for reverse transcription reaction using PrimeScript RT Reagent Kit (Takara, USA). Glyceraldehyde 3-phosphate dehydrogenase (GAPDH) expression was used as an internal control. The information of primers was as follows: Gapdh (forward) 5'-

AACGACCCCTTCATTGAC-3', Gapdh (reverse) 5'-TCCACGACATACTCAGCAC-3', Interleukin-1 $\beta$ (IL-1 $\beta$, forward) 5'-GAAATGCCACCTTTTGACAGTG-3', II-1 $\beta$ (reverse) 5'-TGGATGCTCTCATCAGGACAG-3', Cd80 (forward) 5'-ACCCCCAAC

ATAACTGA GTCT-3', Cd80 (reverse) 5'-TTCCAACCAAGAGAAGCGAG-3'.. Gene expression was analyzed using $2^{(-\triangle \triangle C T)}$ method.

Cells were harvested in a lysis buffer (150 mM NaCl, $50 \mathrm{mM}$ Tris-Cl, $1 \%$ NP-40, 0.5\% DOC, $0.1 \%$ SDS). The membrane was blocked with $4 \%$ non-fat milk in TBST (TBS-Tween 20 solution) for 60 minutes and were incubated with the following antibodies: anti IL-1 $\beta$ (mouse, 1:1000, CST, D6D6T) and CD80 (mouse, 1:1000, Solarbio, K106433P) overnight at $4{ }^{\circ} \mathrm{C}$. The signals were developed using ECL reagent (Amersham). The band intensities were quantified using the Analyze-Gel in image $\mathrm{J}$ software (Wayne Rasband).

Statistical Analysis

The one-way analysis of variance (ANOVA) and Student-Newman-Keuls (SNK) were performed to compare the differences among the groups. Differences were considered statistically significant at pvalue less than 0.05. All statistical analyses were performed using the GraphPad Prism 6 (GraphPad Software, La Jolla, Calif).

\section{Results And Discussion}

\subsection{Active compounds combinatorial filtering}

The satisfactory pharmacokinetic properties are often important indicators for a compound to exert pharmacological or biological activity. Therefore, four in silico ADME models including log P, OB, Caco-2 and $B B B$ were introduced for screening the active compounds from Maotai liquor. Compounds that successfully pass through the $A D M E$ properties criteria of $O B \geq 60 \%, 1 \geq \log P \geq 5$, Caco-2 $\geq-0.4, B B B \geq$ 0.3 were screened as the potential active compounds for further analysis (Table S1).

Consequently, total 55 (accounting for $13.9 \%$ of all 396) bioactive components from Maotai liquor pass through our filter criteria (as shown in Table S1), including terpinen-4-ol $(O B=91.17 \%, \log P=2.55$, Caco$2=1.29$ and $\mathrm{BBB}=1.47), 2$-methylhexanoic acid $(\mathrm{OB}=88.38 \%, \log \mathrm{P}=2.27, \mathrm{Caco}-2=0.88$ and $\mathrm{BBB}=$ 1.09), cedrol $(O B=87.55 \%, \log P=3.16, C a c o-2=1.28$ and $B B B=1.42)$, phthalide $(O B=64.14 \%, \log P=$ 1.44 , Caco-2 $=1.16$ and $B B B=1.28)$, methyl thio furoate $(O B=62.74 \%, \log P=1.64$, Caco-2 $=1.06$ and $B B B=1.27), 2,6-$ dimethoxyphenol $(O B=60.70 \%, \log P=1.53$, Caco- $2=1.23$ and $B B B=1.36)$ and so on. In addition, compound tetramethylpyrazine is also considered as active compound due to its reported 
pharmacology activities [25], although having relatively poor $A D M E$ properties $(O B=29.22 \%, \log P=0.66$, Caco-2 $=1.20$ and BBB $=1.11$ ). These active compounds with suitable ADME properties have the potential to transport into the blood circulation in human body so as to exert pharmacological effects. Interestingly, several screened bioactive compounds have been reported to exhibit extensive biological and pharmacological properties. For instance, cedrol $(\mathrm{OB}=87.55 \%, \log \mathrm{P}=3.16, \mathrm{Caco}-2=1.28$ and $\mathrm{BBB}=$ 1.42 ) is a bioactive sesquiterpenes found in essential oil and shows anti-inflammatory activity, which is commonly used in traditional medicine and cosmetics. Moreover, the evidence showed that benzothiazole has been used as a precursor for pharmacological agents to exert immunomodulatory activity, hencing possible application in autoimmune diseases [26]. The compound tetramethylpyrazine has been reported involved in multiple significant biological functions, especially being used as an inhibitor of phosphodiesterase that contributed to the treatment of cardiovascular diseases in China [25].

In conclusion, 55 compounds from Maotai are filtered out as active entities for the next step of analyzing which are considered to exhibit extensive biological functions and pharmacological activities in the current study. We used combinatorial screening of ADME properties to filter out the most promising molecules of Maotai liquor, building the foundation of a systematic approach for compound selection.

\subsection{Target fishing and analysis}

In order to interpret the potential molecular mechanism of Maotai liquor, target proteins of the active compounds were identified based on integrated in silico approaches, including SEA, STITCH and chemogenomic model, mining the databases of HIT, TTD and DrugBank. Therefore, in this section, a total of 80 potential targets and 385 compound-target interactions (as shown in Table S2) were predicted for 55 active compounds. The results reveal that the majority of active compounds act on multiple biological targets, exhibiting multiple pharmacological mechanisms of the bioactive molecules. For instance, compound ethyl propionate targets on 13 proteins, and terpinen-4-ol can interact with 11 targets.

It is known that Maotai liquor plays a role in various pharmacological effects, including immune regulation, anti-inflammatory, cardiovascular disease, Alzheimer's disease and so on. The targets which are involved in these pharmaceutical processes are potentially therapeutic entities for their related disease. In this light, 80 potential targets were further mapped to PharmGkb, TTD and CTD database to obtain their corresponding diseases. The relationships between these targets and their relevant diseases are shown in Table S2.

To further investigate the meaningful functional annotation of 80 potential targets, Gene Ontology (G0) enrichment analysis were performed. A widely used tool Cytoscape plugin ClueGO was used to effectively implement this procedure for identifying the biological interpretation of large lists of genes in biological networks. As shown in Fig. 2, the results were split into 2 categories: molecular function (GOMF) and biological process (GOBP). In Fig. 2a, it is interesting to observe that the potential targets are involved in a variety of molecular functions including oxidoreductase activity, acting on paired donors, incorporation or reduction of molecular oxygen, adrenergic receptor activity, coenzyme binding, protein phosphatase $2 \mathrm{~A}$ binding and carboxylic ester hydrolase activity, which may participate into the pathophysiology of a 
broad spectrum of human disorders. For GOBP analysis (Fig. 2b), the biological processes of the targets were mainly classified into three categories: circulatory system process, blood circulation and reactive oxygen species metabolic process. These results suggest that the targets were enriched in correlation to the pathogenesis of various diseases including immune diseases, inflammation, cardiovascular disease and Alzheimer's disease.

\subsection{Compound-Target-Disease network construction and analysis}

It seems highly likely that two or more compounds target multiple proteins simultaneously at reasonably close affinities in the biological network which would reach a certain equilibrium to produce a pharmacological effect yet without serious side effects. Significantly, with the increasing understanding of complex diseases, a growing realization that compounds should target comprehensive disease-related network rather than a single protein to get maximum therapeutic effect and minimum adverse effect. Thus, it is imperative to develop network-based method for interpreting the complicated relationships between multi-component and multi-target that are involved in complex diseases at a system level. Fortunately, network pharmacology has emerged as a powerful tool for predicting and analyzing complex systems in pharmacology studies [6]. Particularly, the C-T-D network is widely applicable for interpreting pharmacological mechanism of compound action by employing compounds, targets and diseases.

Although the epidemiology survey result showed that consuming Maotai liquor $150 \mathrm{~g}$ for ten years daily did not cause diseases, but could help protect human health. However, the underlying pharmacological mechanisms of the active compounds in Maotai liquor are still poorly understood. Therefore, in this study, to decipher the complex mechanism of action of active compounds on systematical level, the C-T-D network was constructed by linking 55 compounds, 80 potential targets and 4 types of diseases. The detail information is as follows (Fig. 3, Table S2).

Cardiovascular diseases

Cardiovascular disease (CVD) is a class of diseases used to describe dysfunction of the heart or blood vessels. The brain, kidney and peripheral arterial can all be influenced by CVD and leading to a major cause of morbidity and mortality worldwide. It is suggested that liquor has desirable pharmacological effects on enhancing the function of cardiovascular and circulatory systems. Interestingly, an inspection of our C-T-D network revealed that active compounds in Maotai liquor had satisfactory pharmacological effects on CVD with 33 related targets (Fig. 3, Table S2).

The established network indicated that 34 active compounds have interactions with target protein alcohol dehydrogenase 1C (ADH1C), which has been demonstrated to modify the impact of alcohol intake on the risk of CVD [27]. Nitric oxide synthase, endothelial (NOS3) hit by 19 active compounds is involved in the regulation of vascular smooth muscle tone. NOS3 as a mediator of angiogenesis caused by the production of NO has been reported to play a pivotal role in cardiovascular disease [28]. The serine protease prothrombin(F2)targeted by 15 compounds is responsible for the formation of thrombosis by 
cleaving fibrinogen to form fibrin in the blood coagulation cascade and activates platelet aggregation via protease-activated receptors [29]. The protein sodium-dependent noradrenaline transporter (SLC6A2) hit by 10 compounds is associated with hypertension, the control of which will contribute to lowering blood pressure and reducing the risk of CVD. All the results suggest that the active compounds interacting with these targets may achieve the goal of reducing cardiovascular risk.

\section{Alzheimer's disease}

Alzheimer's disease is a progressive and irreversible brain disease that will result in slowly loss of memory, thinking, and reasoning skill because of the destruction of brain cells, and even eventually the ability to perform the simplest tasks of daily living. Despite great research efforts into the pathophysiology of Alzheimer disease, the complex molecular mechanism underlying Alzheimer disease is still unclear. Previous evidence suggested that moderate daily liquor intake plays a role in the prevention of Alzheimer's disease through reducing the concentration of uric acid in serum [2].

According to the C-T-D network (Fig. 3, Table S2), 19 proteins have been identified related to Alzheimer's disease, such as acetylcholinesterase (ACHE), cathepsin D (CTSD), cholinesterase (BCHE), amine oxidase [flavin-containing] B (MAOB), amine oxidase [flavin-containing] A (MAOA), and peroxisome proliferatoractivated receptor gamma (PPARG). For instance, ACHE identified as the target of 15 compounds in our analysis, is a potential target for the treatment of Alzheimer's disease, inhibitors of which have been developed as first-line drugs in clinical therapy [30]. Butyrylcholinesterase (BCHE) hit by 9 active compounds has been reported to participate in the termination of cholinergic neurotransmission, clarifying it a promising target and diagnosis marker of Alzheimer's disease [31]. In addition, seven active compounds were associated with the target protein Sodium-dependent dopamine transporter (SLC6A3), abnormal expression of which is correlated with genetic susceptibility to Alzheimer's disease. Amine oxidase [flavin-containing] B (MAOB) act as a potential target to the prevention of neurodegeneration in Alzheimer's disease, was found to possess five connected compounds [32]. All these findings reveled that active compounds in Maotai might regulate the nervous system via a complicated compound-protein interaction network, which may shed light on the prevention or treatment of nervous system dysfunction especially Alzheimer's disease.

Immune system disease

The immune system is a complicated network of cells, tissues, and organs that work together to defend the body against foreign substances or dangerous invaders in the environment and maintain overall health. Disorders of the immune system fails to protect the body resulting in recurring and life-threatening infections. It's very important to strengthen and balance our immune system as much as possible, since the human immune system has played a central role in our survival.

As can be seen from the network (Fig. 3, Table S2), 50 compounds connected 9 targets were found to have relationships with the immune system diseases. Among these targets, Trypsin-1 (PRSS1) exhibits the highest number of active compound interactions (46), followed by Dipeptidyl peptidase 4(DPP4)with 
17 compounds, immunoglobulin heavy constant gamma 1 (IGHG1) with 7 compounds, which indicates their significant roles played in the immune system disease. It has been demonstrated that PRSS1 is involved in the regulation of immune response by inhibiting lipopolysaccharide-stimulated toll-like receptor 4 signaling [33]. DPP4 as a multifunctional protein not only plays an important role in T-cell function but also is involved in the pathophysiology of diverse autoimmune diseases. Furthermore, Arginase-1 (ARG1), related to two active compounds, is a key mediator of immune response by regulating availability of arginine [34]. Thus, success in the regulation of these target proteins may be beneficial for the enhancement of immune function so as to improve the health conditions.

\section{Anti-inflammation}

Inflammation is a necessary part of the protective response in body to a foreign challenge or tissue injury, which keeps the normal tissue structure and function by eliminating various deleterious surroundings. Inflammation plays a role in many diseases such as inflammatory bowel disease, cardiovascular disease and autoimmune disorders. Hence, more attention should be paid to develop novel anti-inflammatory agents.

Inspecting the C-T-D networks, 19 inflammation-related targets that link with 48 corresponding active compounds were filtered out (Fig. 3, Table S2). For example, 25 active compounds were identified to bind Alcohol dehydrogenase 1B (ADH1B), which is correlated with inflammation since the abrogation of ADH1B activates the tumor-promoting inflammation in colorectal cancer [35]. Trypsin-3 (PRSS3) targeted by 18 active compounds is related to inflammatory response, inhibitors of which have been shown to play a key role in the treatment of inflammatory gastrointestinal diseases [36]. Carbonic anhydrase I (CA I) as a major cecal bacterial antigen expressed on the surface of intestinal epithelial cells of the colon is related to 14 compounds, which is known to be an effective therapeutic target for the regulation of inflammatory bowel disease. In addition, prostaglandin G/H synthase 2 (PTGS2) hit by 8 active compounds has been demonstrated to play a major role in the pathogenesis of inflammation, control of which can prevent damage of the inflammatory factor in the progression of inflammatory disease [37]. These findings indicate that the multiple interactions between compounds and target proteins might be significant for exerting a pharmacological effect on inflammation.

\subsection{Pathway analysis}

To further understand the potential pharmacological mechanism of Maotai liquor at the pathway level, we mapped the predicted targets onto their related pathways enriched from DAVID database (https://david.ncifcrf.gov/), resulting in 20 canonical pathways including metabolic pathways, neuroactive ligand-receptor interaction, calcium signaling pathway, PI3K-Akt signaling pathway and Tolllike receptor signaling pathway (Fig. 4a). In particular, a T-P network which linked the targets and corresponding signaling pathways was displayed in Fig. 4b.

Through the analysis of our results, the enriched pathways have been uncovered to participate in various biological processes which are mainly associated with four categories, including cardiovascular diseases, 
Alzheimer's disease, immune system disease and inflammation. For example, 11 targets marked in the calcium signaling pathway are involved in the regulation of cardiovascular cells and plays a key role in mediating CVDs by systolicing blood pressure levels. Vascular smooth muscle contraction pathway are also associated with CVDs, inhibition of which blunted the proliferation of intimal vascular smooth muscle cells and is undoubtedly an effective way to take part in the treatment of CVDs [38].

In addition, some canonical pathways are related to neurological diseases, such as metabolic pathways and neuroactive ligand-receptor interaction. As can be seen from Fig. 4, of all 80 target proteins, 26 participate in the metabolic pathways. The metabolism mapping of serum from Alzheimer's patients observed the alterations of metabolites that are involved in multiple metabolic pathways regulating oxidative stress and energy deficiencies and so on, suggesting the pivotal role of metabolic pathways in Alzheimer's disorders [39]. 17 target proteins are involved in mediating the neuroactive ligand-receptor interaction pathway which might provide neuroprotective strategies to Alzheimer's disease. A recent study showed that the enrichment analysis for genetic interactions associated with Alzheimer's disease yielded a number of gene-gene pairs that belong to neuroactive ligand-receptor interaction [40]. Simultaneously, toll-like receptor signaling pathway plays a significant role in the modulation of immune response through recognizing pathogen-associated molecular patterns released by various invading microbes. While cAMP signaling is a major pathway involved in the regulation of immune responses since cAMP is a potential negative regulator of T cell through the induction and activation of PKA (protein kinase A) [41]. For inflammation, PI3K-Akt signaling pathway (involved 8 targets) as a central mediator in signal transduction pathways can mediate the cellular proliferation and survival, as well as negatively regulate the expression of the LPS-induced inflammation [42].

In summary, our results indicated that multiple target proteins could act on multiple pathways to exert their specific pharmacological effects at the pathway level, including regulating the pathologic processes of cardiovascular diseases, Alzheimer's disease, immune system disease and inflammation.

\subsection{Experimental validation}

To validate the reliability of our approach, the anti-inflammatory pharmacological effects and immune regulation of several active compounds in Maotai liquor were explored using in vitro cell models.

For anti-inflammation, mRNA and protein levels of IL-1 $\beta$ were detected in ANA-1 macrophage treated with LPS and indicated chemicals. The real-time PCR results showed that LPS stimulated a 90 -fold increase of $\|-1 \beta$ expression $(p<0.0001)$ validating the inflammatory response in ANA-1 cell line. Intriguingly, both of the candidate compounds, cedrol and benzothiazole, significantly inhibited IL-1 $\beta$ mRNA expression in a dose dependent manner. The cedrol $(10 \mu \mathrm{g} / \mathrm{mL})$ and benzothiazole $(20 \mu \mathrm{g} / \mathrm{mL})$ significantly suppressed the expression of $I 1-1 \beta$ by $74.5 \%(p<0.01)$ and $42.8 \%(p<0.05)$ respectively (Fig. $5 a)$. The antiinflammatory effects of these two candidates were also confirmed on protein level in the whole cell lysates. ANA-1 cells pretreated with cedrol $(10 \mu \mathrm{g} / \mathrm{mL})$ and benzothiazole $(20 \mu \mathrm{g} / \mathrm{mL})$ showed significantly decreased expression of IL1- $\beta$ by about $67.3 \%(p<0.001)$ and $44.7 \%(p<0.01)$ compared with LPS treatment group respectively (Fig. 5b). 
In addition, we examined the immunomodulatory role of tetramethylpyrazine and 3-pentanol using the immature dentric cell line DC2.4. The expression of costimulatory molecule CD80 was used to evaluate the immune response to LPS. The real-time PCR results showed that the addition of LPS stimulated over 2 -fold increase of $C d 80$ expression ( $p<0.01$ ), which was significantly repressed by the treatment of tetramethylpyrazine and 3-pentanol in a dose dependent manner. Tetramethylpyrazine $(10 \mu \mathrm{g} / \mathrm{mL})$ and 3pentanol $(20 \mu \mathrm{g} / \mathrm{mL})$ decreased the LPS induced Cd80 expression by about $78 \%(p<0.01)$ and $55 \%(p<$ 0.05 ) respectively (Fig. 6a). Furthermore, the western blot analysis suggested the inhibitory effects of these two candidate compounds on the raised expression of CD 80 by LPS. Similarly, we observed the $57.6 \%(p<0.001)$ and $43.7 \%(p<0.01)$ decrease in DC 2.4 cells treated with tetramethylpyrazine and 3pentanol compared with LPS treatment group, respectively (Fig. 6b).

These data showed that cedrol and benzothiazole are likely responsible for anti-inflammatory effects, while tetramethylpyrazine and 3-pentanol have potential in immune regulation. The obtained results suggested that our approach was reasonable to verify the pharmacological effect of the compounds in Maotai liquor, so as to contribute to clarifying the relationship between Maotai liquor and public health.

\section{Conclusion}

Maotai liquor regarded as one of the most famous distillates in the world has been consumed for a very long time because of its good taste and healthy aspects. However, the pharmacological mechanisms of complex compounds in Maotai liquor still remains unclear.

In this study, a systematic framework is proposed by integrating in silico ADME evaluation, multi target prediction, GO enrichment analysis, network pharmacology technology, pathway analysis, as well as experimental verification to clarify the pharmacological effects and mechanism of flavor compounds in Maotai liquor. The major findings are concluded as follows: 1) 55 components out of 391 compounds with satisfactory pharmacokinetic profiles in Maotai liquor are identified as active substances by the combinatorial evaluation for $\mathrm{OB}$, log $\mathrm{P}, \mathrm{caco}-2$ and BBB. 2) The screened active molecules may interact with single or multiple target proteins involved in various diseases which provides a broader perspective on the pharmacological mechanisms of active compounds in Maotai liquor. 3) The GO enrichment analysis is performed for molecular function and biological process to provide significant biological functional annotation of our obtained targets. 4) The constructed compound-target-disease network further clarifies the pharmacological effects of multiple components in Maotai liquor from a holistic perspective. 5) The pathway analysis is applied to map the targets involved in the biological pathways so as to understand the pharmacological mechanisms perturbed by multiple targets at the pathway level. 6) The in vitro experiment is performed to verify the reasonability of our strategy. Four compounds including cedrol and benzothiazole, tetramethylpyrazine and 3-pentanol are confirmed to play a role in antiinflammatory and immune regulation respectively.

In summary, we proposed an integrative strategy by using systems pharmacology to investigate the potential active compounds and their related targets, as well as to understand the potential pharmacological mechanism of flavor compounds in Maotai liquor. The present work will not only shed 
light on the mechanism of active compounds in Maotai liquor at the system level, but also provide a novel approach for discovery of the active compounds that may benefit to human health.

\section{Declarations}

\section{Conflict of interest}

The authors declare no conflict of interest.

\section{Acknowledgements}

The research is supported by the Shenzhen City Science and technology program (No.SW201110010) and Shenzhen City Key project of science and technology program (No.JCYJ20140418095735538).

Author Contributions

W. Zhou and Z.G. Liu devised the study. W. Zhou and G.H. Zhang conducted data preparation. W. Zhou and Z.Y. Chen drafted the manuscript, and revised it critically. W. Zhou, Z.Y. Chen and P. C. Y carried out experiments and conducted data analysis. All authors approved the final version of the manuscript and agreed to be accountable for all aspects of the work.

\section{References}

1. Huang F, Wang H: Analysis on the relationship between Chinese liquor and health. Liquor Making 2013, 40:14-19.

2. Fan $\mathrm{W}, \mathrm{Xu} \mathrm{Y}$ : Review of functional factors and quality safety factors of baijiu (Chinese liquor). LiquorMaking Science \& Technology 2012, 3:002.

3. Wu J, Cheng M-L, Zhang G-H, Zhai R-W, Huang N-H, Li C-X, Luo T-Y, Lu S, Yu Z-Q, Yao Y-M: Epidemiological and histopathological study of relevance of Guizhou Maotai liquor and liver diseases. World J Gastroentero/ 2002, 8(3):571.

4. Yi X, Long L, Yang C, Lu Y, Cheng M: Maotai ameliorates diethylnitrosamine-initiated hepatocellular carcinoma formation in mice. PLoS One 2014, 9(4):e93599.

5. Zhou W, Wang Y: A network-based analysis of the types of coronary artery disease from traditional Chinese medicine perspective: potential for therapeutics and drug discovery. J Ethnopharmacol 2014, 151(1):66-77.

6. Zhou W, Wang J, Wu Z, Huang C, Lu A, Wang Y: Systems pharmacology exploration of botanic drug pairs reveals the mechanism for treating different diseases. Scientific reports 2016, 6:36985.

7. Fan W, Xu Y, Qian MC: Identification of aroma compounds in Chinese "Moutai" and "Langjiu" liquors by normal phase liquid chromatography fractionation followed by gas

chromatography/olfactometry. In: Flavor chemistry of wine and other alcoholic beverages. ACS Publications; 2012: 303-338. 
8. Li H, Lian B, Ding Y, Nie C, Zhang Q: Bacterial diversity in the central black component of Maotai Daqu and its flavor analysis. Annals of microbiology 2014, 64(4):1659-1669.

9. Zhu S, Lu X, Ji K, Guo K, Li Y, Wu C, Xu G: Characterization of flavor compounds in Chinese liquor Moutai by comprehensive two-dimensional gas chromatography/time-of-flight mass spectrometry. Anal Chim Acta 2007, 597(2):340-348.

10. Xiao Z, Yu D, Niu Y, Chen F, Song S, Zhu J, Zhu G: Characterization of aroma compounds of Chinese famous liquors by gas chromatography-mass spectrometry and flash GC electronic-nose. Journal of Chromatography B 2014, 945:92-100.

11. Kim J-S, Kam SF, Chung HY: Comparison of the volatile components in two Chinese wines, Moutai and Wuliangye. Journal of the Korean Society for Applied Biological Chemistry 2009, 52(3):275-282.

12. Van De Waterbeemd H, Gifford E: ADMET in silico modelling: towards prediction paradise? Nature reviews Drug discovery 2003, 2(3).

13. Arnott JA, Planey SL: The influence of lipophilicity in drug discovery and design. Expert opinion on drug discovery 2012, 7(10):863-875.

14. Tetko IV, Bruneau P: Application of ALOGPS to predict 1-octanol/water distribution coefficients, logP, and $\log \mathrm{D}$, of AstraZeneca in-house database. Journal of pharmaceutical sciences 2004, 93(12):3103-3110.

15. Lipinski CA, Lombardo F, Dominy BW, Feeney PJ: Experimental and computational approaches to estimate solubility and permeability in drug discovery and development settings. Advanced drug delivery reviews 2012, 64:4-17.

16. Barnes PM, Bloom B, Nahin RL: Complementary and alternative medicine use among adults and children; United States, 2007. 2008.

17. Xu X, Zhang W, Huang C, Li Y, Yu H, Wang Y, Duan J, Ling Y: A novel chemometric method for the prediction of human oral bioavailability. International journal of molecular sciences 2012, 13(6):6964-6982.

18. Awortwe C, Fasinu P, Rosenkranz B: Application of Caco-2 cell line in herb-drug interaction studies: current approaches and challenges. Journal of pharmacy \& pharmaceutical sciences: a publication of the Canadian Society for Pharmaceutical Sciences, Societe canadienne des sciences pharmaceutiques 2014, 17(1):1.

19. Pham The H, González-Álvarez I, Bermejo M, Mangas Sanjuan V, Centelles I, Garrigues TM, CabreraPérez MÁ: In Silico Prediction of Caco-2 Cell Permeability by a Classification QSAR Approach. Molecular informatics 2011, 30(4):376-385.

20. Li L, Li Y, Wang Y, Zhang S, Yang L: Prediction of human intestinal absorption based on molecular indices. J Mol Sci 2007, 23:286-291.

21. Kam A, M Li K, Razmovski-Naumovski V, Nammi S, Chan K, Li Y, Q Li G: The protective effects of natural products on blood-brain barrier breakdown. Current medicinal chemistry 2012, 19(12):18301845. 
22. Li L, Li Y, Wang Y, Zhang S: Prediction of BBB permeation based on molecular indices. Chinese journal of medicinal chemistry 2007, 17(4):221.

23. Yu H, Chen J, Xu X, Li Y, Zhao H, Fang Y, Li X, Zhou W, Wang W, Wang Y: A systematic prediction of multiple drug-target interactions from chemical, genomic, and pharmacological data. PloS one 2012, 7(5):e37608.

24. Smoot ME, Ono K, Ruscheinski J, Wang P-L, Ideker T: Cytoscape 2.8: new features for data integration and network visualization. Bioinformatics 2010, 27(3):431-432.

25. Liao M-H, Wu C-C, Yen M-H: Beneficial effects of tetramethylpyrazine, an active constituent of Chinese herbs, on rats with endotoxemia. Proceedings of the National Science Council, Republic of China Part B, Life sciences 1998, 22(1):46-54.

26. Khan KM, Mesaik MA, Abdalla OM, Rahim F, Soomro S, Halim SA, Mustafa G, Ambreen N, Khalid AS, Taha M: The immunomodulation potential of the synthetic derivatives of benzothiazoles: Implications in immune system disorders through in vitro and in silico studies. Bioorganic chemistry 2016, 64:21-28.

27. Latella MC, Di Castelnuovo A, De Lorgeril M, Arnout J, Cappuccio FP, Krogh V, Siani A, Van Dongen M, Donati MB, De Gaetano G: Genetic variation of alcohol dehydrogenase type 1C (ADH1C), alcohol consumption, and metabolic cardiovascular risk factors: results from the IMMIDIET study. Atherosclerosis 2009, 207(1):284-290.

28. Wang L, Shen C, Yang S, Chen Y, Guo D, Jin Y, He L, Chen J, Zhao X, Zhao H: Association study of NOS3 gene polymorphisms and hypertension in the Han Chinese population. Nitric Oxide 2015, 51:16.

29. Lee K, Park CW, Jung W-H, Park HD, Lee SH, Chung KH, Park SK, Kwon OH, Kang M, Park D-H: Efficacious and orally bioavailable thrombin inhibitors based on a 2, 5-thienylamidine at the P1 position: discovery of $\mathrm{N}$-carboxymethyl-d-diphenylalanyl--prolyl [(5-amidino-2-thienyl) methyl] amide. Journal of medicinal chemistry 2003, 46(17):3612-3622.

30. Cortes N, Sierra K, Alzate F, Osorio EH, Osorio E: Alkaloids of Amaryllidaceae as Inhibitors of Cholinesterases (AChEs and BChEs): An Integrated Bioguided Study. Phytochemical Analysis 2017.

31. Košak U, Brus B, Knez D, Žakelj S, Trontelj J, Pišlar A, Šink R, Jukič M, Živin M, Podkowa A: The Magic of Crystal Structure-Based Inhibitor Optimization: Development of a Butyrylcholinesterase Inhibitor with Picomolar Affinity and In Vivo Activity. Journal of medicinal chemistry 2017.

32. Košak U, Knez D, Coquelle N, Brus B, Pišlar A, Nachon F, Brazzolotto X, Kos J, Colletier J-P, Gobec S: NPropargylpiperidines with naphthalene-2-carboxamide or naphthalene-2-sulfonamide moieties: Potential multifunctional anti-Alzheimer's agents. Bioorganic \& medicinal chemistry 2017, 25(2):633645.

33. Komatsu H, Shimose A, Shimizu T, Mukai Y, Kobayashi J, Ohama T, Sato K: Trypsin inhibits lipopolysaccharide signaling in macrophages via toll-like receptor 4 accessory molecules. Life Sci 2012, 91(3-4):143-150. 
34. Zou Y, Chen Q, Ye Z, Li X, Ju R: VEGFR1 Signaling Regulates IL-4-Mediated Arginase 1 Expression in Macrophages. Current molecular medicine 2017, 17(4):304-311.

35. Villéger R, Beswick E, Johnson P, Qiu S, Powell D, Pinchuk I: P-183 Abrogation of alcohol dehydrogenase-1B expression by $\mathrm{CD} 90+$ stromal cells supports tumor-promoting inflammation in colorectal cancer. Annals of Oncology 2016, 27(suppl_2):ii54-ii54.

36. Rolland-Fourcade C, Denadai-Souza A, Cirillo C, Lopez C, Jaramillo JO, Desormeaux C, Cenac N, Motta J-P, Larauche M, Taché Y: Epithelial expression and function of trypsin-3 in irritable bowel syndrome. Gut 2017:gutjnl-2016-312094.

37. Rumzhum N, Ammit A: Cyclooxygenase 2: its regulation, role and impact in airway inflammation. Clinical \& Experimental Allergy 2016, 46(3):397-410.

38. Li X, Xu X, Wang J, Yu H, Wang X, Yang H, Xu H, Tang S, Li Y, Yang L: A system-level investigation into the mechanisms of Chinese Traditional Medicine: Compound Danshen Formula for cardiovascular disease treatment. PLoS One 2012, 7(9):e43918.

39. González-Domínguez R, García-Barrera T, Gómez-Ariza JL: Metabolite profiling for the identification of altered metabolic pathways in Alzheimer's disease. Journal of pharmaceutical and biomedical analysis 2015, 107:75-81.

40. Meda SA, Koran MEl, Pryweller JR, Vega JN, Thornton-Wells TA: Genetic interactions associated with 12-month atrophy in hippocampus and entorhinal cortex in Alzheimer's Disease Neuroimaging Initiative. Neurobiology of aging 2013, 34(5):1518. e1519-1518. e1518.

41. Wehbi VL, Taskén K: Molecular mechanisms for cAMP-mediated immunoregulation in T cells-role of anchored protein kinase A signaling units. Frontiers in immunology 2016, 7:222.

42. Guha M, Mackman N: The phosphatidylinositol 3-kinase-Akt pathway limits lipopolysaccharide activation of signaling pathways and expression of inflammatory mediators in human monocytic cells. Journal of Biological Chemistry 2002, 277(35):32124-32132.

\section{Figures}
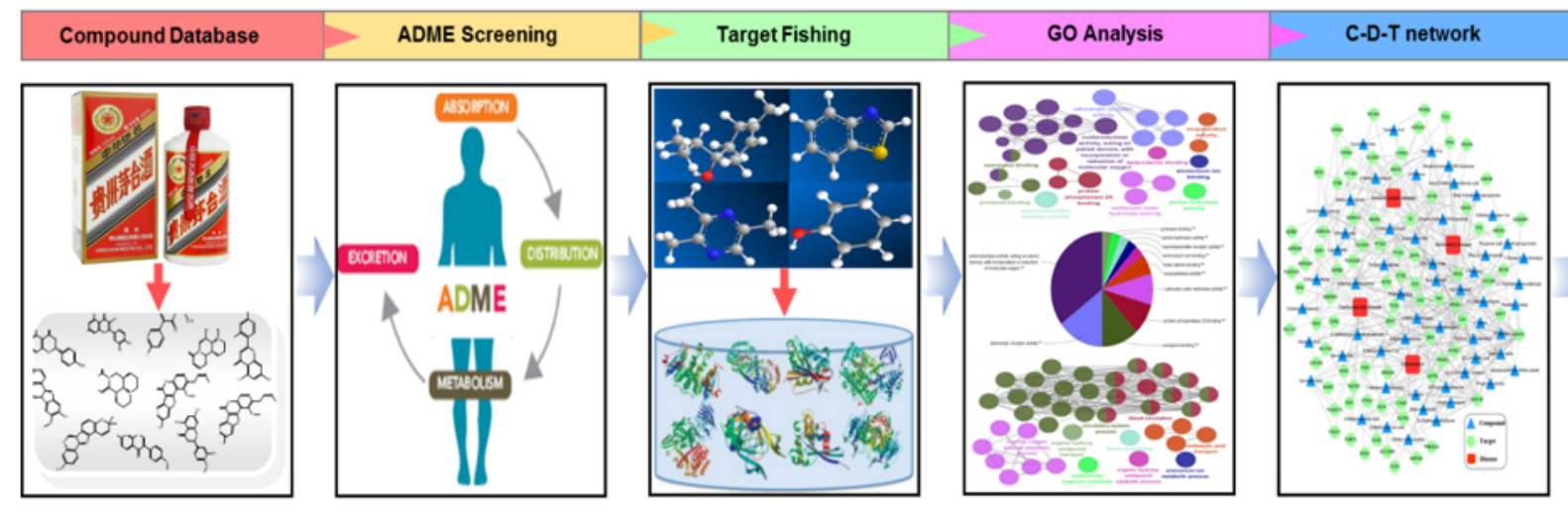
Pathway Analysis

Figure 1 
The framework of systems pharmacology approach.
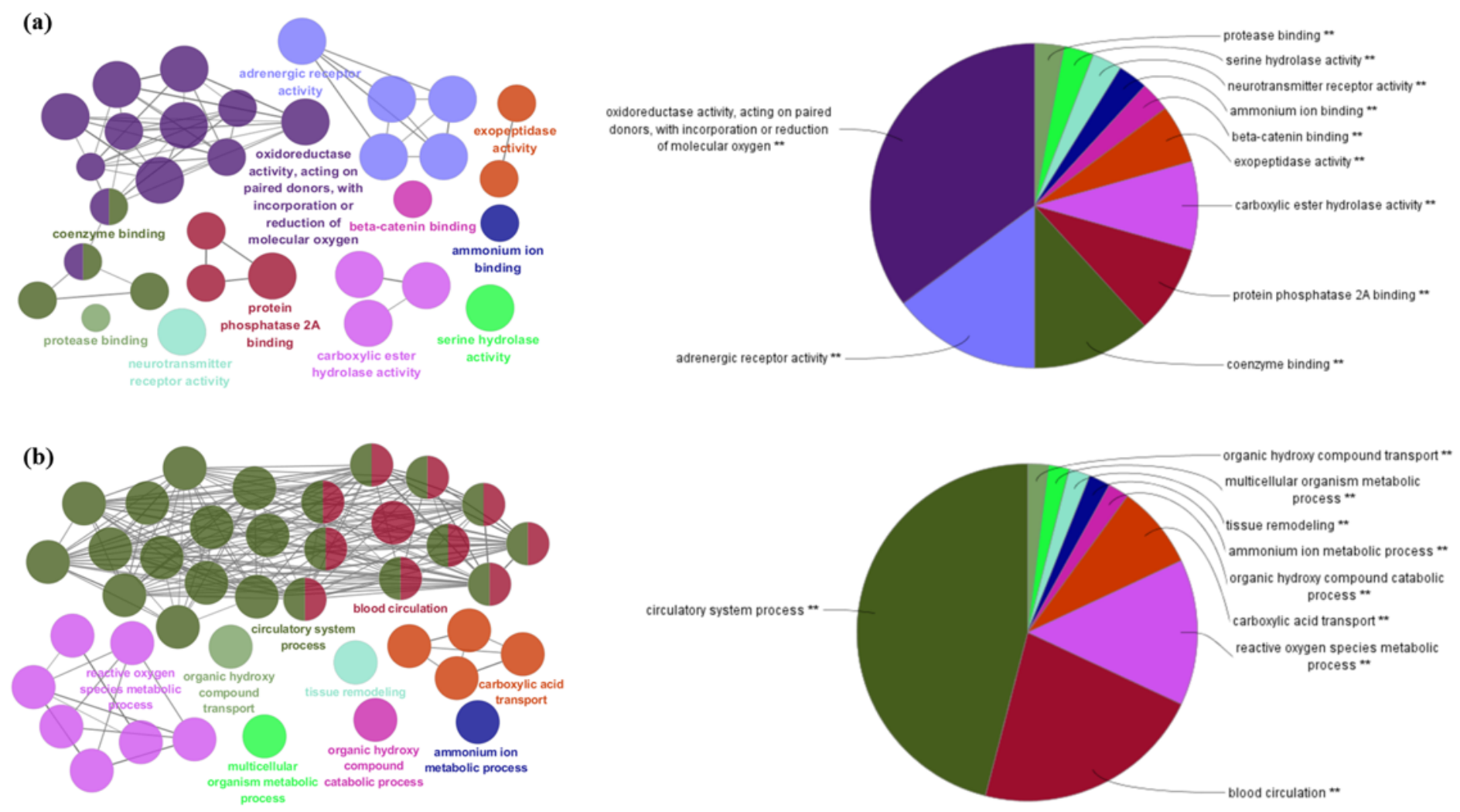

\section{Figure 2}

GO analysis of the predicted targets. GO terms are represented as nodes, and the node size represents the term enrichment significance. The node pie charts represent the molecular function and biological process. Only the most significant term per group is labeled. (a) Representative molecular function interactions among targets. (b) Representative biological processes interactions among targets. 


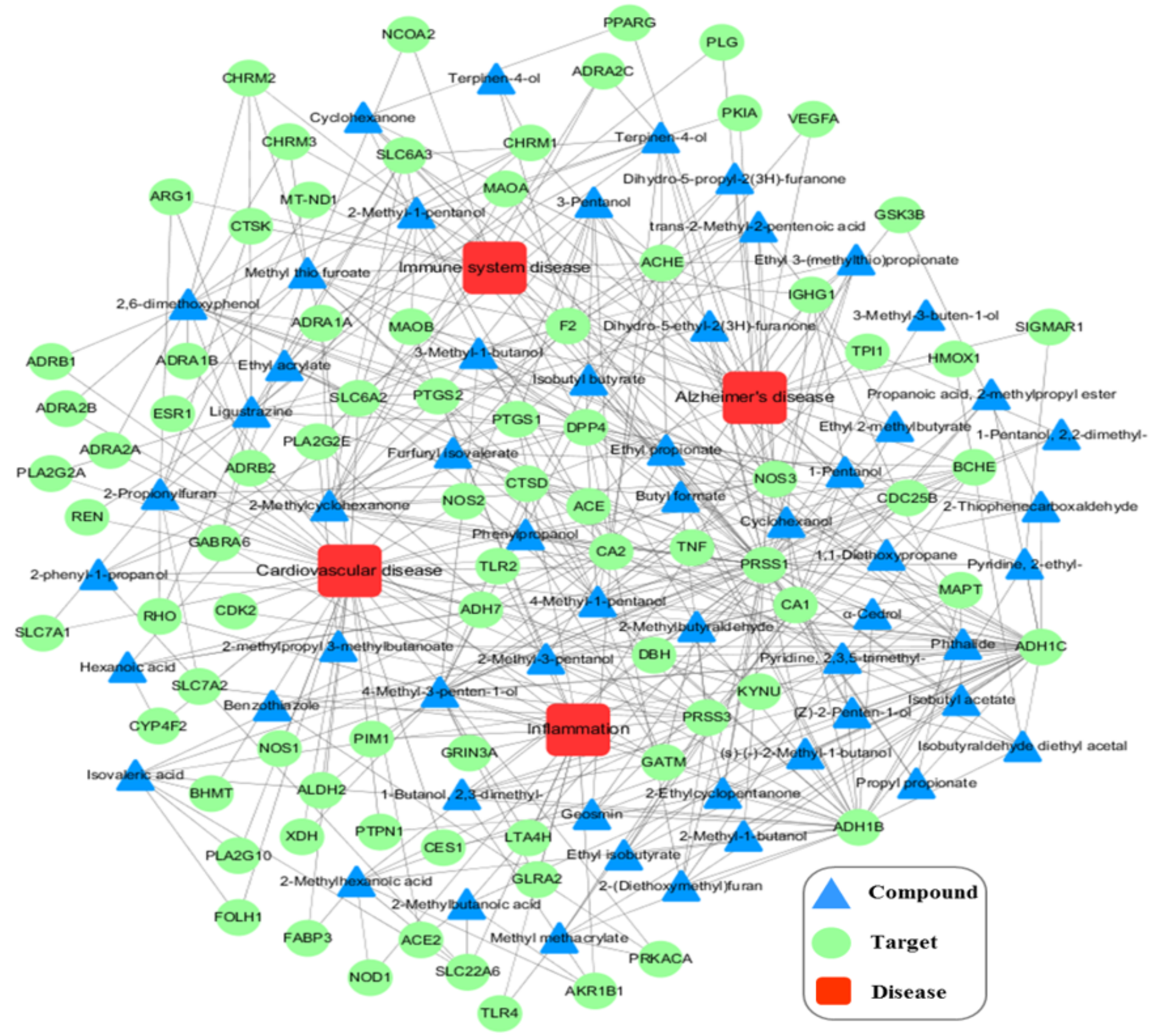

Figure 3

C-T-D network of Maotai liquor. 


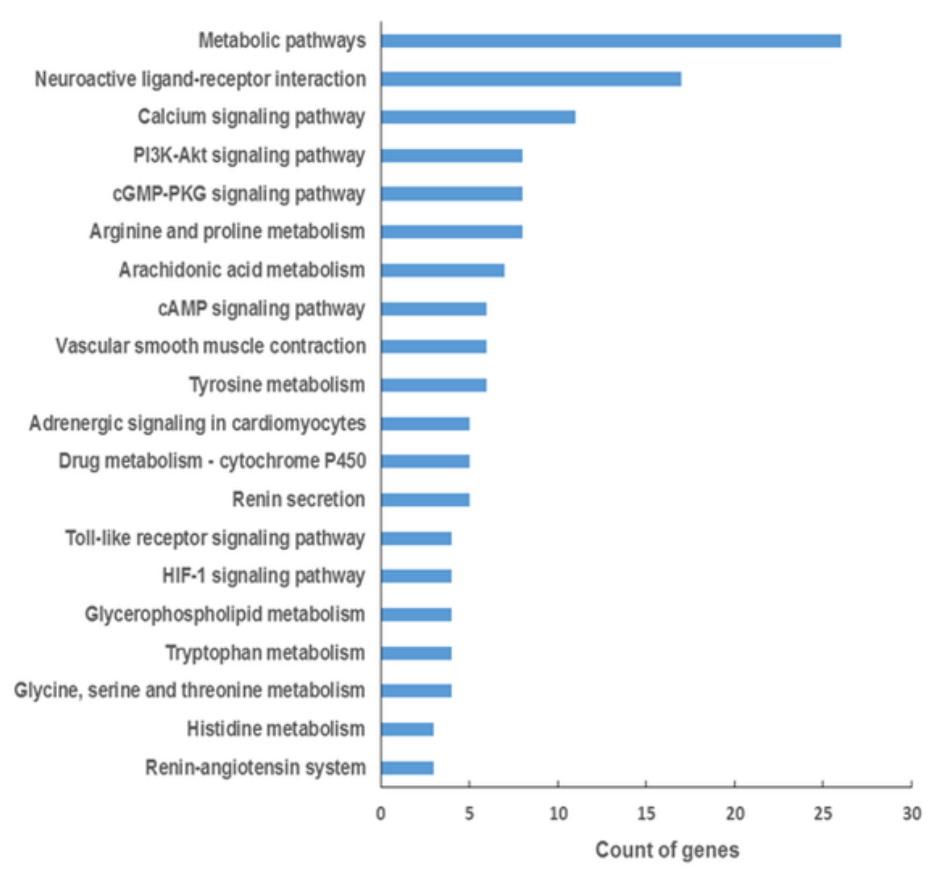

(a)

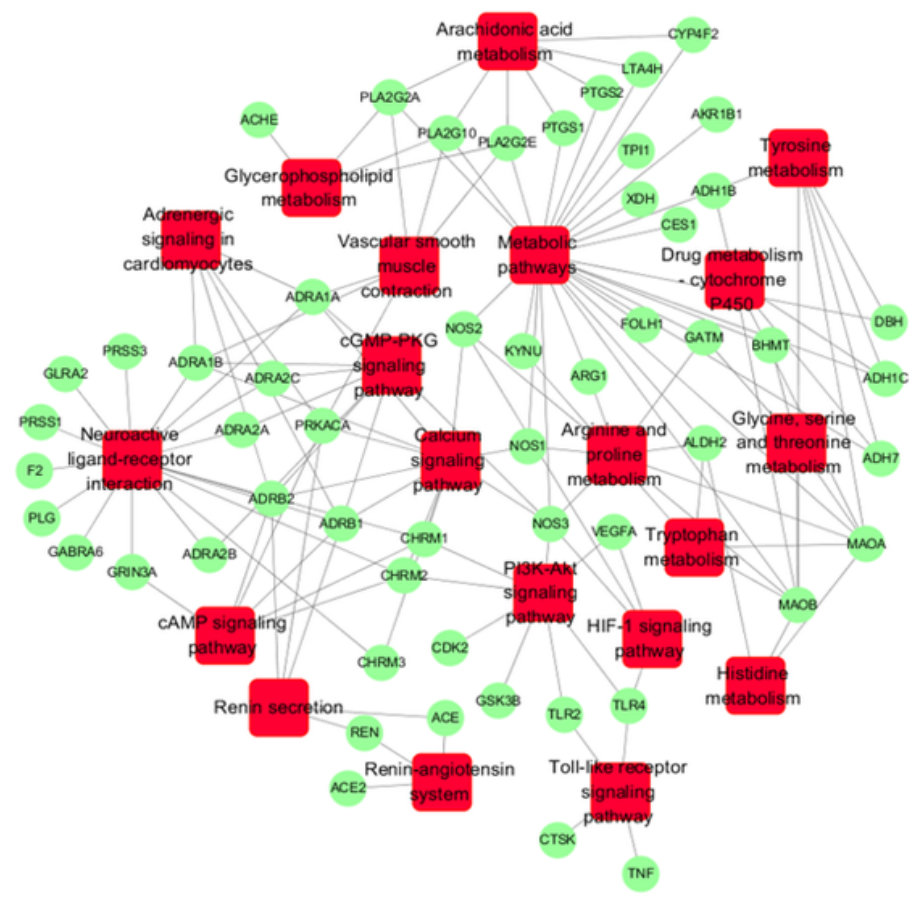

(b)

\section{Figure 4}

(a) KEGG pathway analysis of the potential targets. The $y$-axis represented the name of significantly enriched canonical pathways relative to the target genes, and $x$-axis represented the counts of targets. (b) Target-Pathway network of active ingredients in Maotai for related diseases. 
(a)

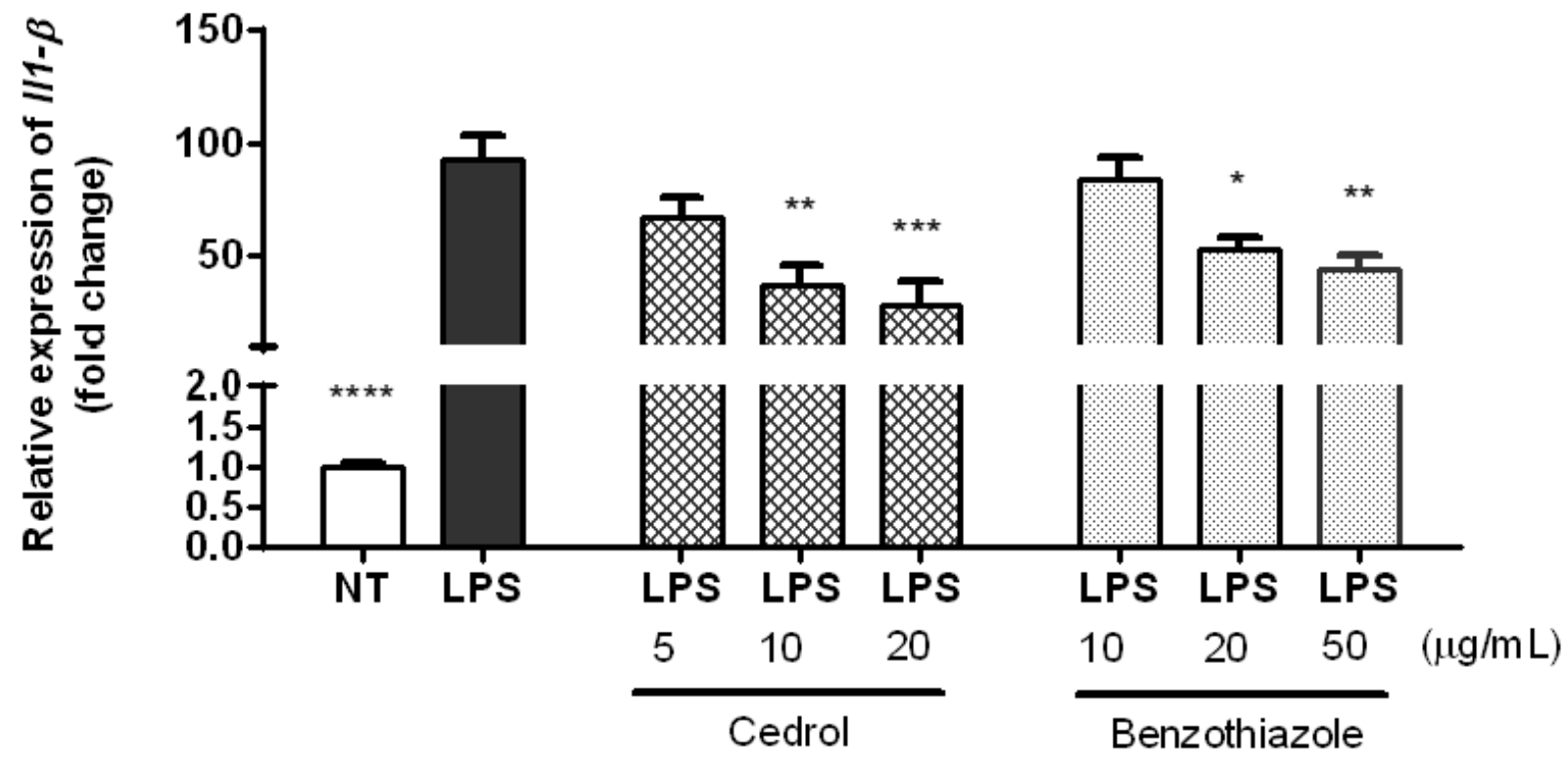

(b)

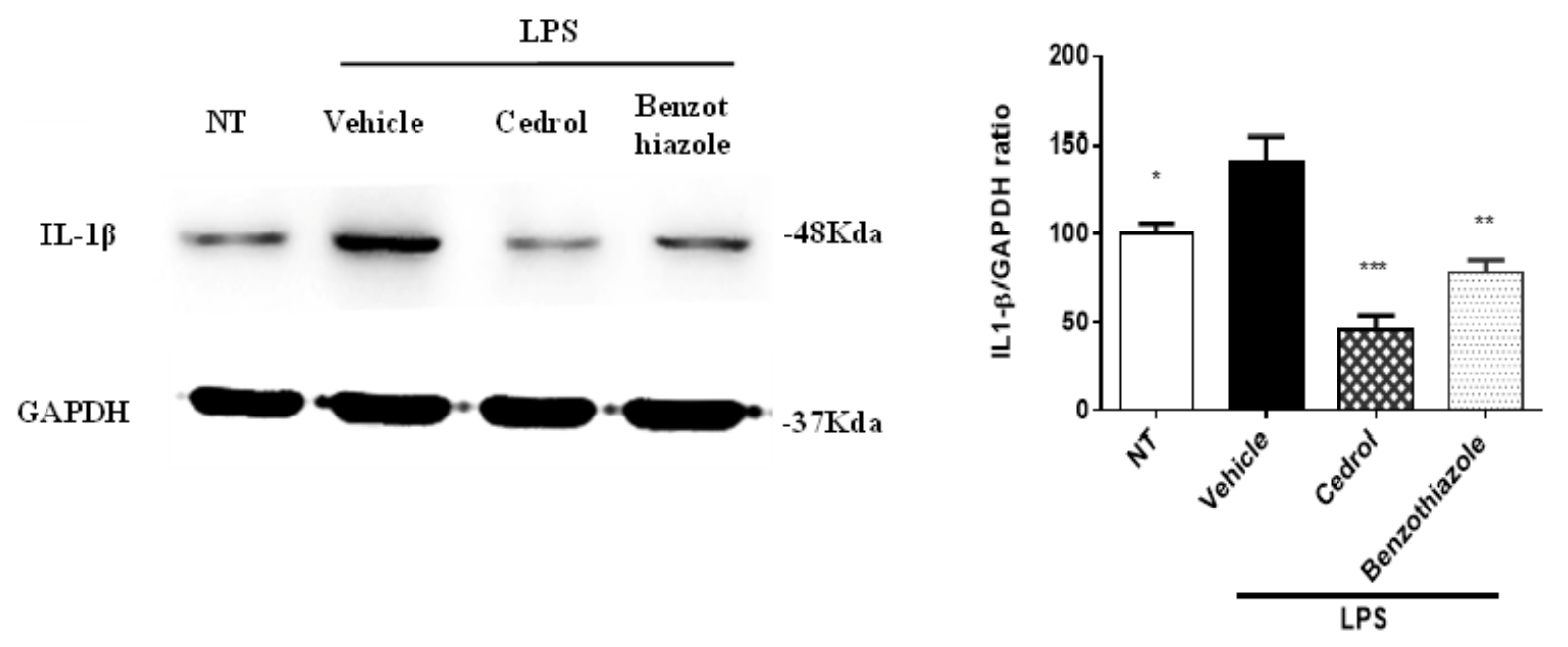

Figure 5

The role of candidate compounds in anti-inflammation. (a) Real-time PCR results showed the expression of II- $1 \beta$ in ANA- 1 cells that are pretreated with various concentrations of cedrol $(5,10,20 \mu \mathrm{g} / \mathrm{mL})$ and benzothiazole $(10,20,50 \mu \mathrm{g} / \mathrm{mL})$. NT indicated control group without treatment. (b) Representative immunoblots and corresponding quantitation showed the expression of IL-1 $\beta$ in ANA-1 cell line. DMSO was used as vehicle control. Cells were cultured with cedrol $(10 \mu \mathrm{g} / \mathrm{mL})$ and benzothiazole $(20 \mu \mathrm{g} / \mathrm{mL})$ individually with the existence of LPS for 24 hours. Quantifications were shown on the right. Data are presented as mean $\pm S E M,{ }^{*}<0.05,{ }^{*} P<0.01, * \star * P<0.001, * \star \star * P<0.0001(n=3$ independent experiments). 
(a)

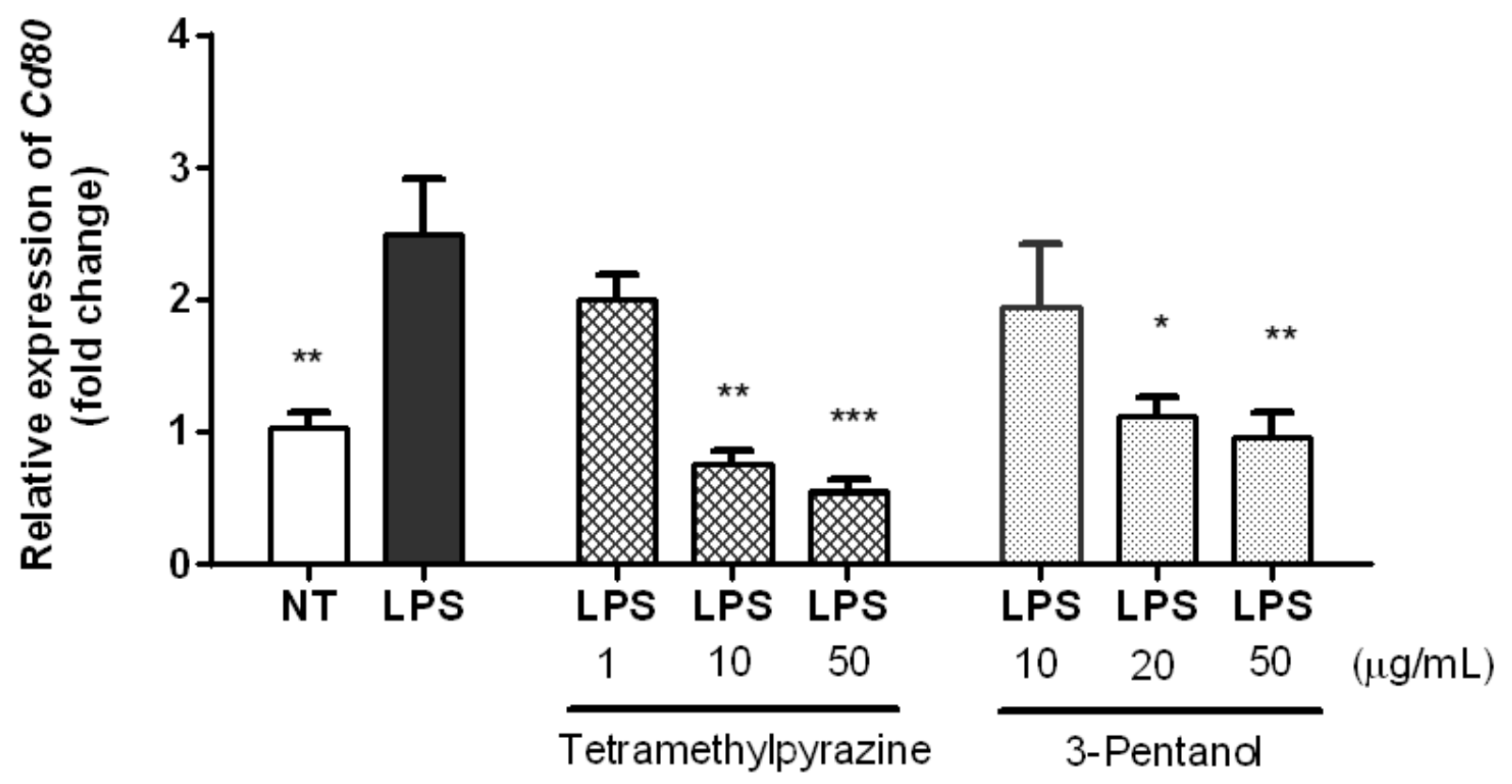

(b)

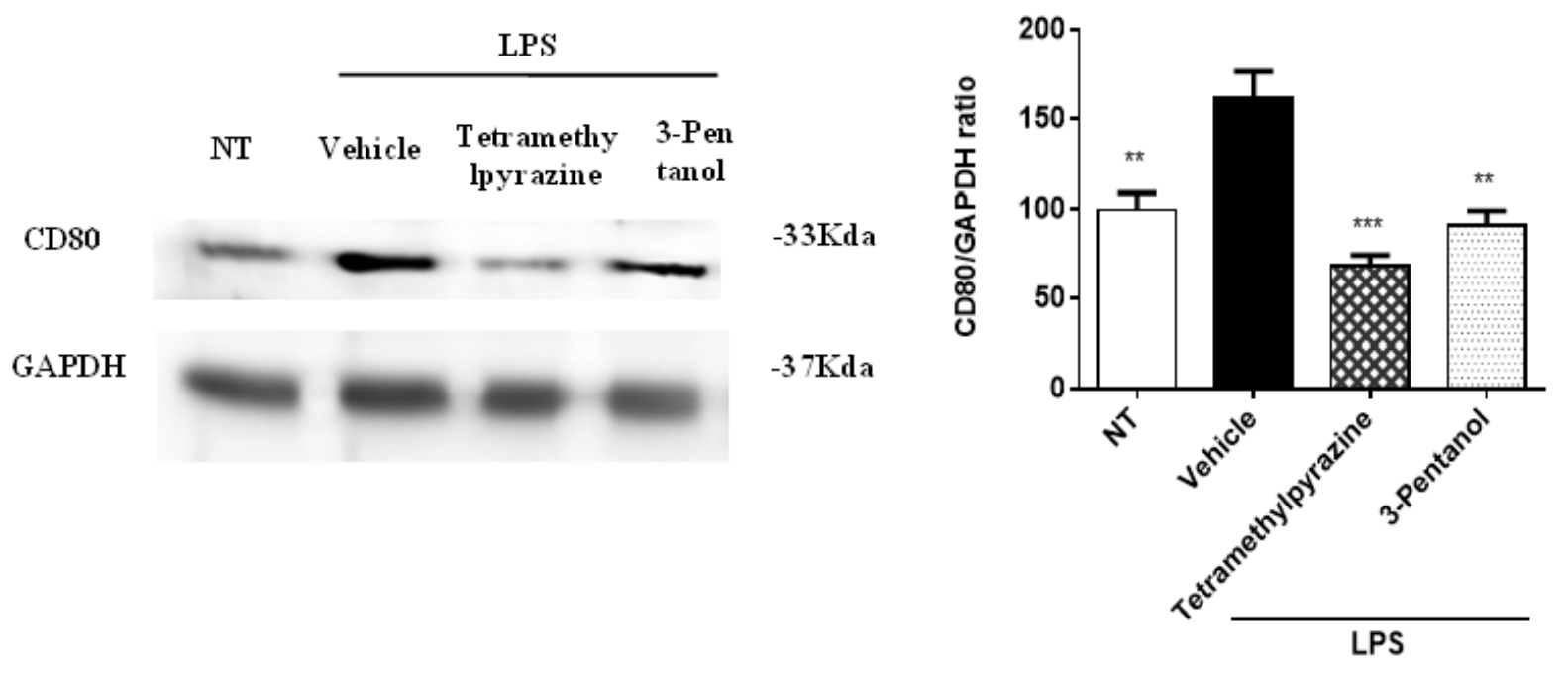

Figure 6

The role of candidate compounds in immune regulation. (a) DC2.4 cells were pretreated with various concentrations of tetramethylpyrazine $(1,10,50 \mu \mathrm{g} / \mathrm{mL})$ and 3-pentanol $(10,20,50 \mu \mathrm{g} / \mathrm{mL})$ with LPS treatment. The expression of Cd80 was examined by real-time PCR. (b) Representative immunoblots and corresponding quantitation showed the expression of CD80 in DC2.4 cell line that was pretreated with tetramethylpyrazine $(10 \mu \mathrm{g} / \mathrm{mL})$ and 3-pentanol $(20 \mu \mathrm{g} / \mathrm{mL})$ and stimulated by LPS for 24 hours. DMSO was used as vehicle control. Quantifications were shown on the right. Data are mean $\pm S E M,{ }^{*} P<0.05$, $\star * P<0.01,{ }^{* \star *} P<0.001$ ( $n=3$ independent experiments).

\section{Supplementary Files}


This is a list of supplementary files associated with this preprint. Click to download.

- TableS2.xlsx

- TableS1.xlsx 